\title{
Application Article \\ Mutual Coupling Effects Analysis in a Cross-Rhombic Antenna Array
}

\author{
Jorge Sosa-Pedroza, Luis Carrión-Rivera, Sergio Peña-Ruiz, and Fabiola Martínez-Zuñiga \\ Departamento de Telecomunicaciones, Sección de Pogrado, Escuela Superior de Ingeniería Mecánica y Eléctrica, \\ Instituto Politécnico Nacional, Unidad Profesional Adolfo López Mateos, Av. Instituto Politécnico Nacional, Edif. Z-4 3er. piso, \\ Col. Lindavista, 07738 Mexico City, DF, Mexico
}

Correspondence should be addressed to Jorge Sosa-Pedroza, jsosa@ipn.mx

Received 8 June 2011; Revised 15 December 2011; Accepted 15 December 2011

Academic Editor: Hon Tat Hui

Copyright ( 12012 Jorge Sosa-Pedroza et al. This is an open access article distributed under the Creative Commons Attribution License, which permits unrestricted use, distribution, and reproduction in any medium, provided the original work is properly cited.

\begin{abstract}
We present an analysis of mutual coupling effects on radiation pattern and individual coupling in a conformal array of cross rhombic antennas. Analysis is made using both full-wave simulation and numerical approaches implemented in Matlab. The array consists of a truncated hexagonal pyramid, with a cross rhombic antenna in each pyramidal face, including the one on the top, having a 7-antennas-array. Results of radiation pattern and S11 parameters are presented, showing mutual coupling effects among the elements.
\end{abstract}

\section{Introduction}

As is well known, antenna arrays are used to improve gain and/or field pattern, difficult to achieve with a single radiator element. Since more than 60 years, the research in the field of antenna arrays has been widely investigated [1-3]. But most of the analysis methods do not consider mutual effects, generated by interaction between antenna elements forming an array. Interaction is known as mutual coupling and is produced by near-field effects which modify the impedance of individual antenna and then the radiated power of each element.

The far-field array analysis, using the field superposition of elements by means of a vector sum, assumes that there are no mutual effects, although the near-field effect modifies the desired far field. So it is necessary to know the near-field performance of an antenna placed in an array. This paper presents analysis of mutual coupling effects of cross-rhombic antenna arrays for both near-field effects (coupling) and farfield effects (field pattern).

First we describe the Cross-Rhombic Antenna used in this analysis, and then we present simulation analysis for linear and conformal array configurations affecting individual impedance and field pattern. Finally we present radiation pattern of the conformal array proposed.

\section{The Cross-Rhombic Antenna}

The Cross-Rhombic Antenna (CRA) is our own design [47]; it is a circularly polarized travelling wave antenna derived from Roederer's cross antenna [8]. We have obtained up to $15 \mathrm{dBi}$ gain and $2 \mathrm{~dB}$ axial ratio, in a $1.6 \lambda$ square area. The CRA is proposed to be used on board of a small satellite and as a receiving antenna for ground-based augmentation systems (GBAS).

The CRA and its radiation pattern are shown in Figure 1; earlier we have performed a parametric analysis [4-7] in order to determine the design procedure of the antenna, and now we analyze its performance as a part of an array, particularly into a conformal array for GPS-L1 (1560$1588 \mathrm{MHz}$ ) band reception.

\section{Mutual Coupling Analysis}

Our first analysis is a small linear array with 8 CRA elements, as shown in Figure 2.

Separation between antenna centers is $1.57 \lambda$, and separation between the closest points in two consecutive antennas is $0.31 \lambda$. Full-wave simulation analysis of S-parameter matrix for the GPS-L1 band is shown in Figure 3. 


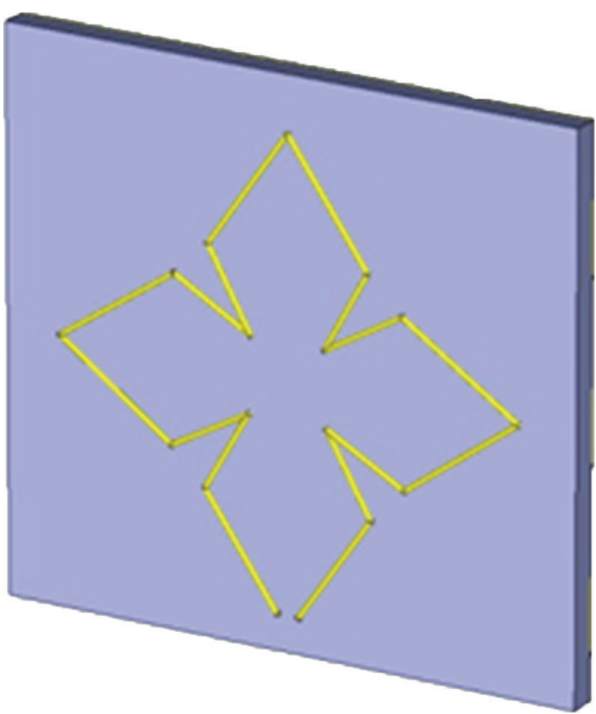

(a)
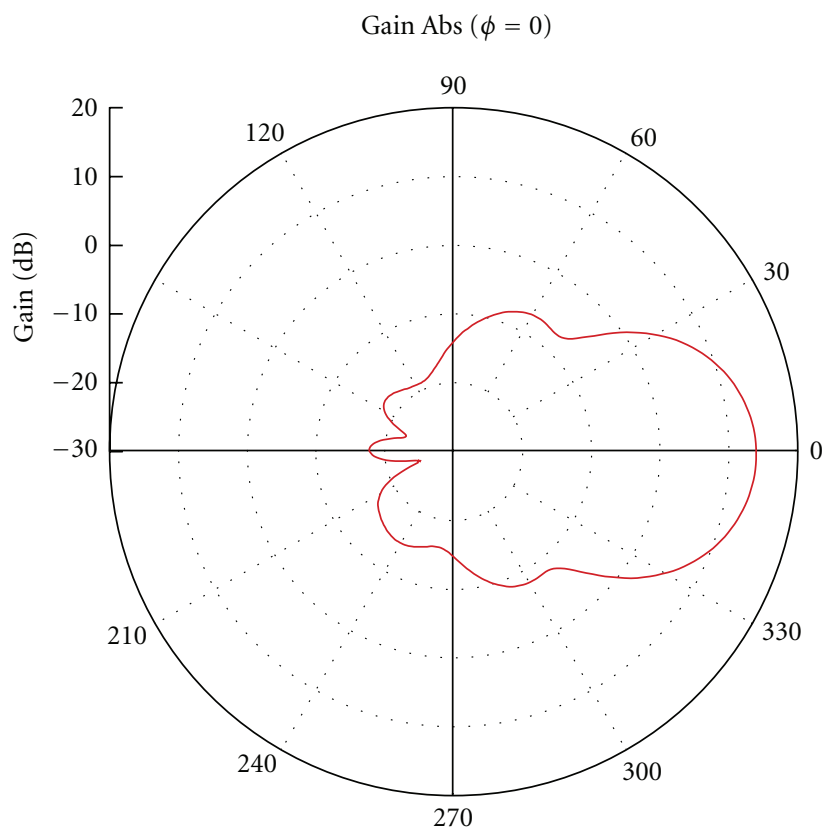

— Gain $(\mathrm{dB})$

Figure 1: (a) The Cross-Rhombic Antenna and (b) its radiation pattern ( $\phi=0^{\circ}$ cut).

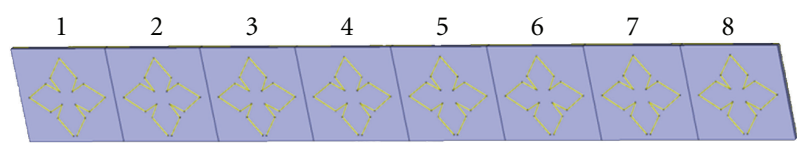

FIGURE 2: Eight-element linear antenna array.

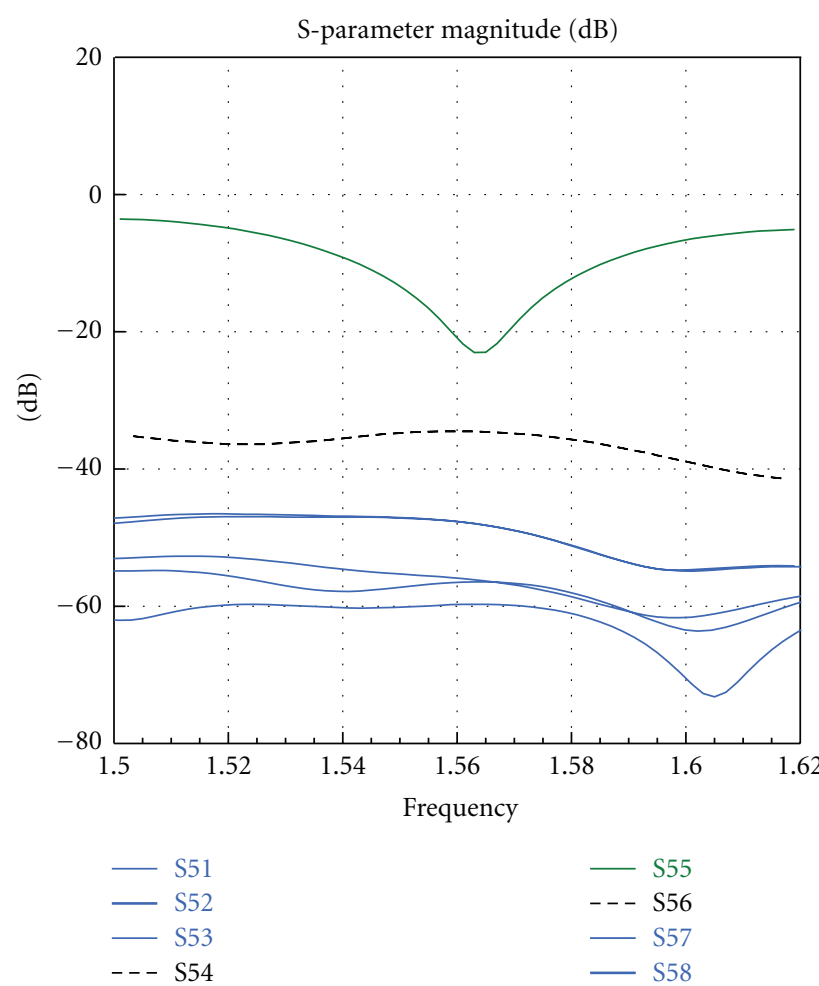

(a)

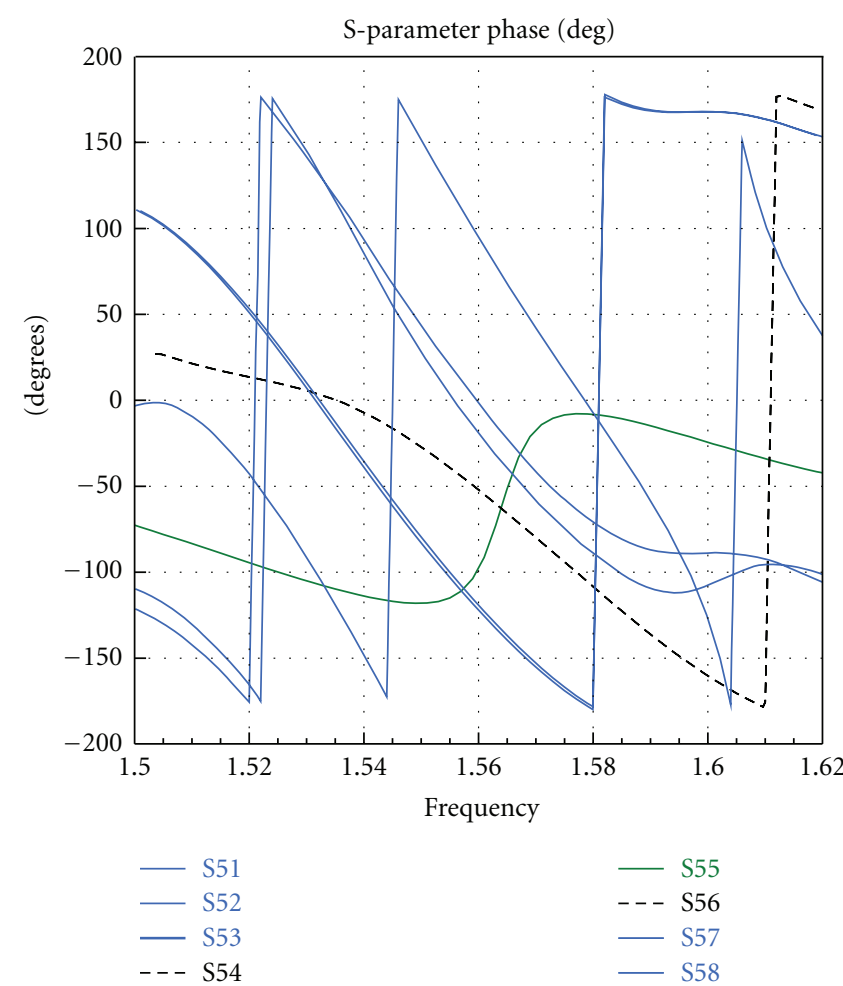

(b)

Figure 3: S-parameter matrix in (a) magnitude (dB) and (b) phase (degrees). 


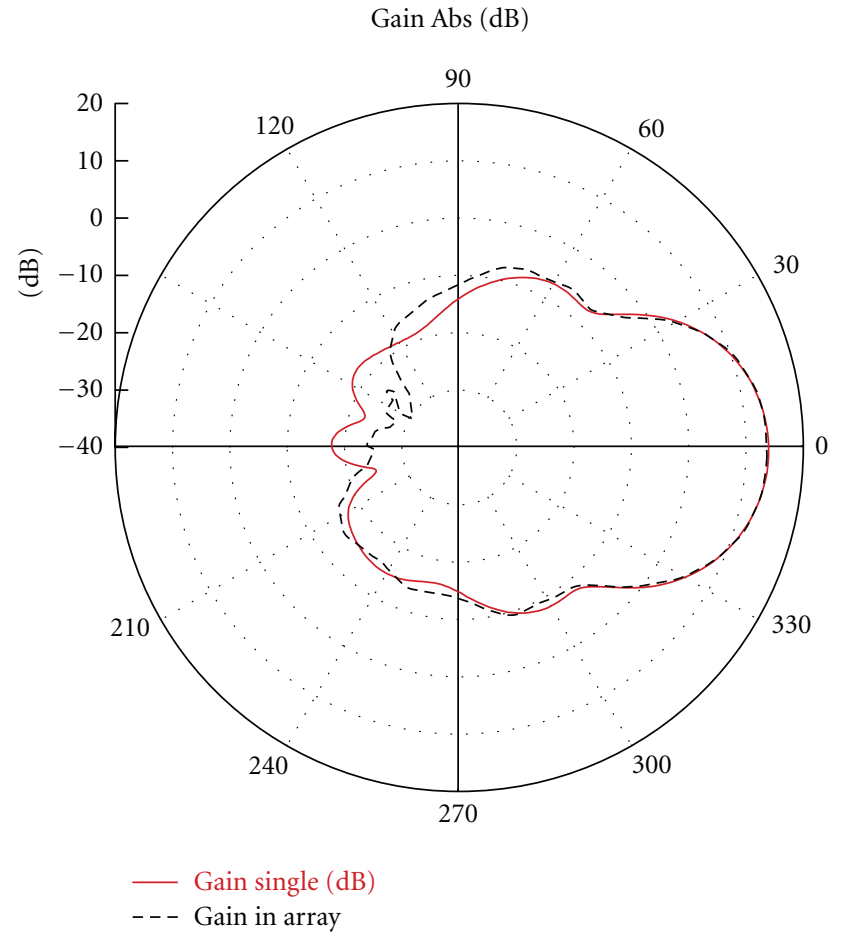

Figure 4: Comparison between isolated (solid-red) and the 5th array element (dashed-brown) radiation patterns.

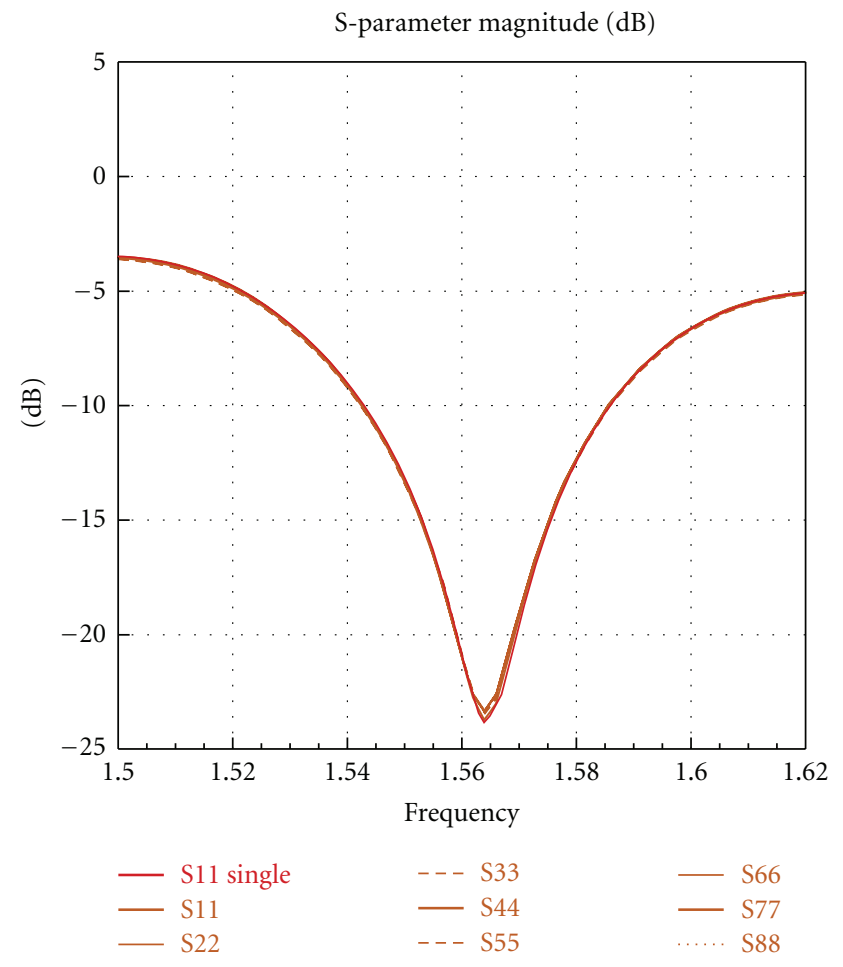

(a)

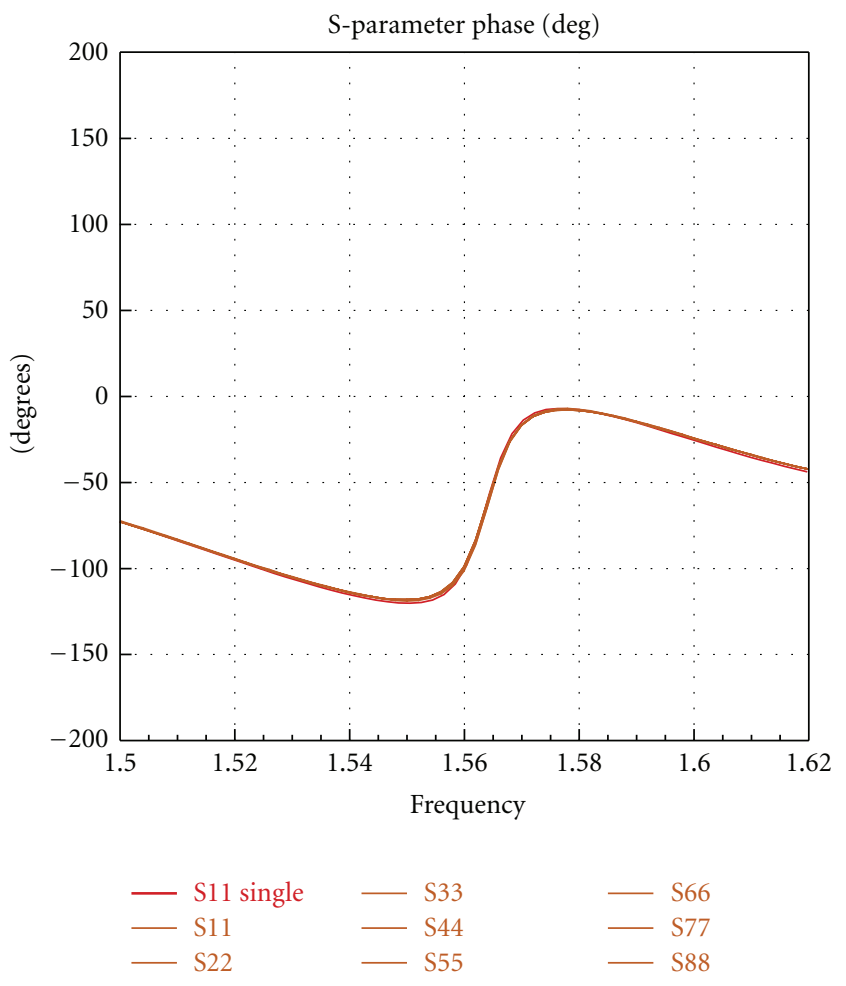

(b)

FIgURE 5: Mutual coupling effects over the array elements: (a) magnitude (dB) and (b) phase (deg). 
Figure 3(a) depicts the S11 magnitude for an isolated element in green solid-line curve, to compare with effects of the array. Blue-colored solid-line curves show mutual effects for all 8 elements, and black-dotted curve is the worst case, corresponding for two elements close one to each other. In the same way, Figure 3(b) depicts the S11 phase parameter and black-dotted curve shows the worst case. Black-dotted curve corresponds to any two close elements; that is, if we take the 5th element as reference, black-dotted curve represents S45 and S65 parameters, showing a value below $-34 \mathrm{~dB}$. Field pattern comparison is shown in Figure 4 for both an isolated element and the 5th element affected by mutual effects. It can be noticed that radiation pattern does not change significantly for the CRA array. On the other hand, Figure 5 shows magnitude and phase S11 parameter comparison between antenna array and the isolated element. We conclude from Figures 3, 4, and 5 that mutual effects for a linear CRA array can be neglected in both cases: individual coupling and individual radiation pattern.

\section{Conformal Antenna Array}

We propose a conformal antenna array (CAA) for application on GPS satellite reception; we are trying to get an almost hemispherical pattern in order to look as most of GPS satellites as possible using a medium gain antenna. There are many approaches to hemispherical coverage [911], even for GPS applications [12] however, they consider that a $-10 \mathrm{dBi}$ gain is well suitable because the use of low noise amplifiers (LNA). We proposed a CAA for a ground based augmentation system (GBAS) needing an all-around high level reception from horizon to zenith. Original idea proposes an antenna capable of providing at least $10 \mathrm{~dB}$ gain over the whole hemisphere. Then we consider the use of a CRA, because of its almost $15 \mathrm{~dB}$ directivity.

\section{Conformal Antenna Array Analysis}

There is a lack of tools for analysis and simulation of conformal antenna arrays. Even though there exists special software providing full-wave conformal simulation, these commercial products are in general very expensive. The EM simulator we have used is CST, and the proposed array is shown in Figure 6(a); it is a hexagonal-truncated pyramid, having one element in each face, including the top face.

Figure 6(b) depicts the conformal array $S_{n n}$ (each element's coupling) response in brown and red solid-line curves and mutual coupling parameters $\left(\mathrm{S}_{m n}\right)$ in blue solid-line and black-dotted curves; as shown the worst case of mutual coupling is around $-40 \mathrm{~dB}$ meaning that mutual effects are so small that they could be neglected, then, results on $S_{n n}$ are non-consistent because frequency sliding of individual coupling in most of the elements means a strong mutual effect; we conclude that CST is not suitable for use in conformal arrays; then, if we want to obtain CAA parameters, we have to use another simulation tool or technique.

Brégains et al. [13] developed a numerical simulation that deals with problems as the one we have; it is called conformal pow 3D (CP3D). We used this free-license

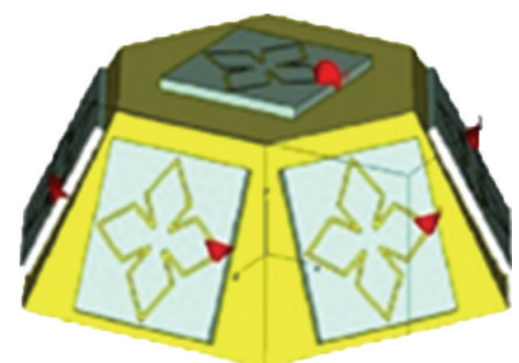

(a)

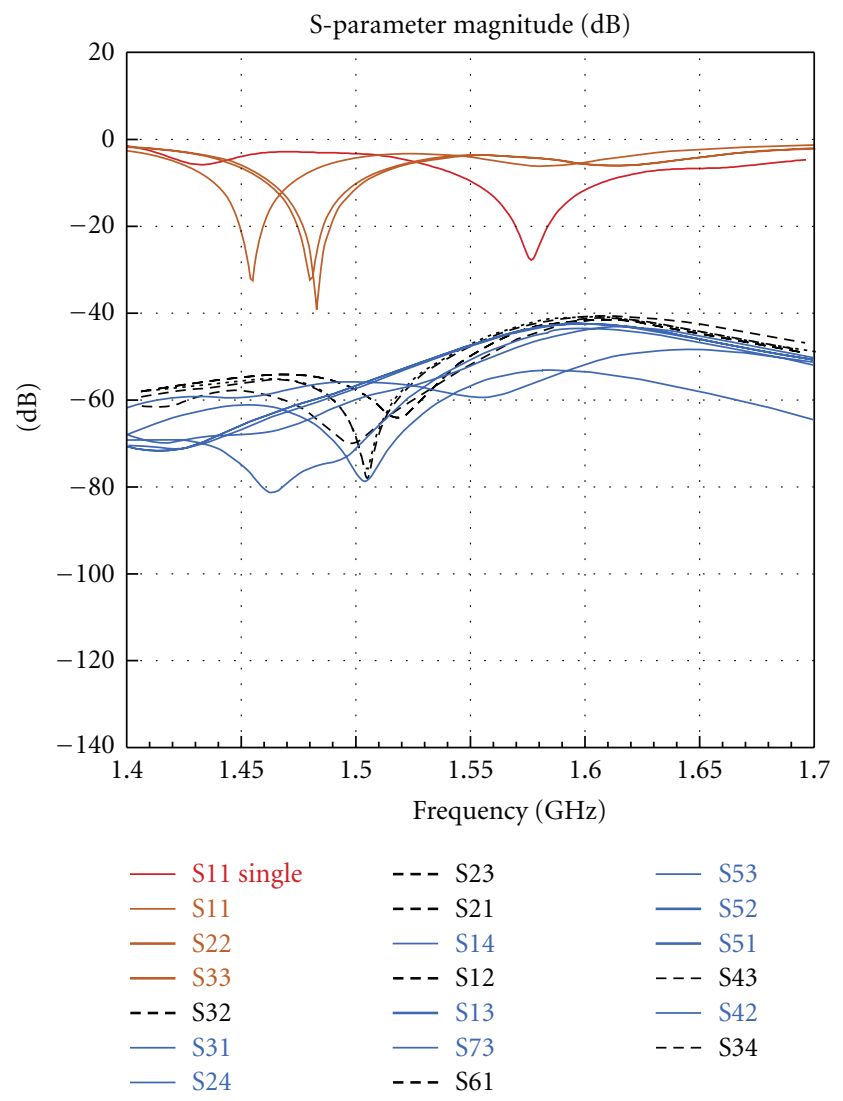

(b)

Figure 6: (a) Hexagonal pyramid conformal array and (b) its Sparameters.

Matlab-based software in order to analyze the radiation pattern of our conformal array. To do so, we have to perform a prior analysis in order to see if there is any special behavior of our antenna when it is placed in an array. As seen in Figures 4 and 5, it is clear that there is a negligible mutualcoupling effect in a linear array; we can expect that mutual effect will be even lower when antennas are not in the same plane, as shown in the lower curves of Figure 6; then, we can neglect mutual coupling in a conformal array.

\section{Computation of the Radiation Pattern of the Conformal Array}

Assuming the fact that the mutual coupling is null because of the CRA and array geometry, we can use conformal pow 3D, modifying only some code lines of the program, in order to 


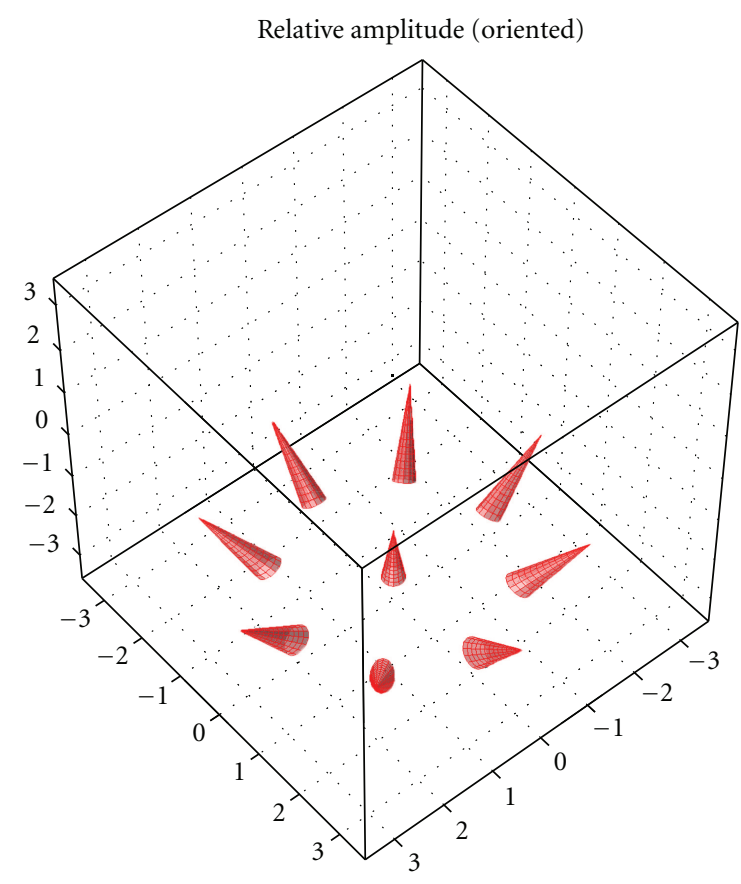

Figure 7: Array elements oriented along each local $Z_{n}$ axis.

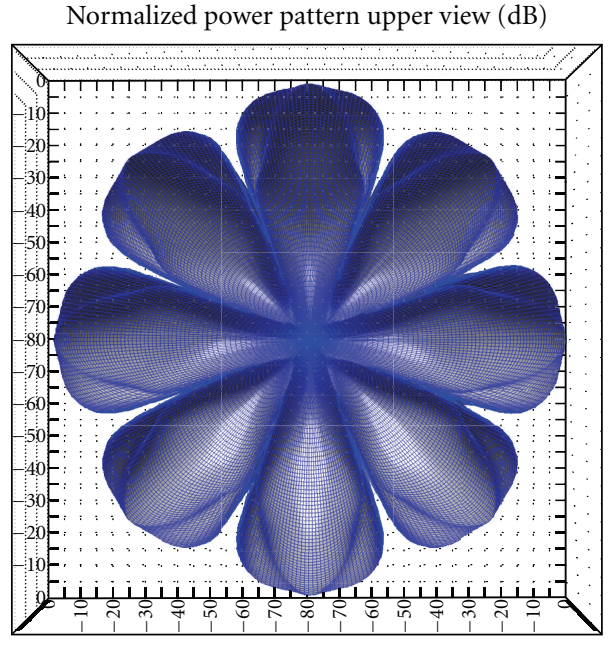

(a)

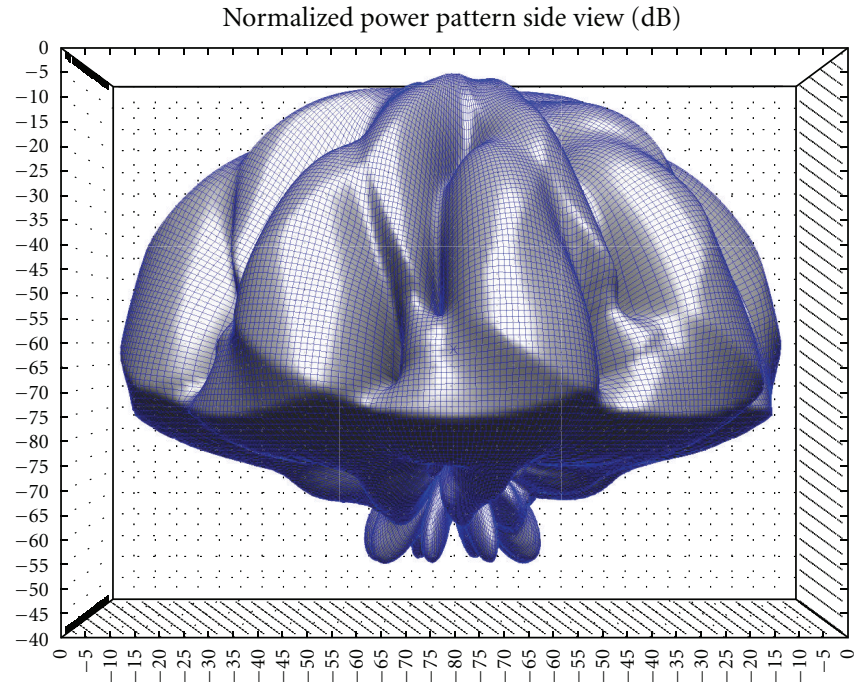

(b)

Figure 8: (a) Upper view and (b) side view of the 3D polar radiation pattern.

include available numerical data of the CRA (imported from text file of the full-wave simulation software).

Figure 7 shows a sketch of CAA, using cones to represent each CRA orientation along its local $Z_{n}$ axis. Using these geometries, the conformal pow $3 \mathrm{D}$ gives results depicted in Figure 8 , showing a 3D polar radiation pattern from two views, and Figure 9 shows the principal-plane cuts for the same radiation pattern.

Former figures show a good agreement between simulation design and proposal gain and field pattern. Coverage of $10 \mathrm{~dB}$ gain over bandwidth is good enough, even if we have some ripple at the center. Large attenuation at each side of $\pm 90^{\circ}$ angle ensures low interference noise, generated by ground reflections.

\section{Conclusions}

We have presented simulation analysis of a conformal antenna array, intended to be used in a GBAS; the proposed antenna for array is a CRA, where analysis shows very low mutual coupling effects, considering its high directivity and very small side lobes. 


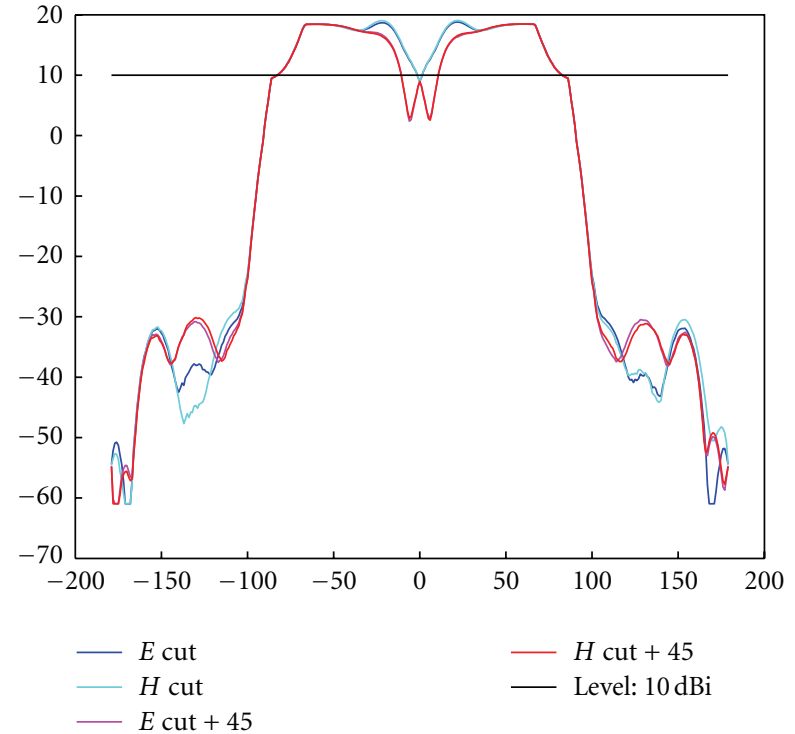

FIGURE 9: Principal-plane cuts of the array radiation pattern.

Considering most commercial simulators are not suitable for use in analysis of CAA, as is shown in this paper, we have used the free-license CP3D, taking in account the low mutual coupling effects for both linear and conformal arrays. Using this computational tool, it was possible to properly deal with our problem.

\section{References}

[1] S. A. Schelkunoff, "A Mathematical theory of linear arrays," Bell System Technical Journal, vol. 22, no. 1, pp. 80-107, 1943.

[2] E. C. Jordan, Electromagnetic Waves and Radiating Systems, Prentice Hall, New York, NY, USA, 2nd edition, 1968.

[3] H. Yagi, "Beam Transmission of ultra-short waves," Proceedings of the IRE, vol. 16, pp. 715-740, 1928.

[4] J. Sosa-Pedroza et al., "Análisis teórico de una Antena rómbica planar de cuatro brazos usando diferentes sustratos," in Proceedings of the 12th Congreso Nacional de Ingeniería Electromecánica y de Sistemas (CNIES '10), pp. 883-888, México, Mexico, November 2010.

[5] J. Sosa-Pedroza et al., "Análisis Teórico Experimental de una Antena Rómbica Planar de Cuatro Brazos," in Proceedings of the 6th International Conference on ElectromechanicsAnd Systems Engineering (CIIES '11), pp. 115-119, México, Mexico, November 2011.

[6] J. Sosa-Pedroza et al., "Parametric analysis of a cross rhombic antenna," in Proceedings of the 1st Workshop on Analog and Digital Electronic Design (WADED '11), pp. 86-89, Guadalajara, Mexico, October 2011.

[7] J. Sosa-Pedroza et al., "Optimización de una Antena Rómbica de Cruz," in Proceedings of the 22th Reunion Internacional de Otoño de Comunicaciones, Computación, Electrónica, Automatización, Robótica y Exposición Industrial (ROC\&C '11), Acapulco, Mexico, December 2011.

[8] A. G. Roederer, "Cross antenna: a new low-profile circularly polarized radiator," IEEE Transactions on Antennas and Propagation, vol. 38, no. 5, pp. 704-710, 1990.

[9] C. N. Hu, C. S. Chuang, D. C. Chou, K. J. Liu, and C. I. Hung, "Design of the cross-dipole antenna with near-hemispherical coverage in finite-element phased array by using genetic algorithms," in Proceedings of the International Conference on Phased Array Systems and Technology, pp. 303-306, Dana Point, Calif, USA, May 2000.

[10] P. Kunott, "Design of a triple patch antenna element for doublecurved conformal antenna arrays," in Proceedings of the 1st European Conference on Antennas and Propagation (EuCAP '06), Nice, France, November 2006.

[11] R. Baggen, M. Martínez-Vázquez, J. Leiss, S. Holzwarth, L. S. Drioli, and P. de Maagt, "Low profile GALILEO antenna using EBG technology," IEEE Transactions on Antennas and Propagation, vol. 56, no. 3, pp. 667-674, 2008.

[12] J. R. Lambert, C. A. Balanis, and D. DeCarlo, "Spherical cap adaptive antennas for GPS," IEEE Transactions on Antennas and Propagation, vol. 57, no. 2, pp. 406-413, 2009.

[13] J. C. Brégains, J. A. García-Naya, A. Dapena, and M. GonzálezLópez, "A MATLAB tool for visualizing the 3D polar power patterns and excitations of conformal arrays," IEEE Antennas and Propagation Magazine, vol. 52, no. 4, Article ID 5638255, pp. 127-133, 2010. 

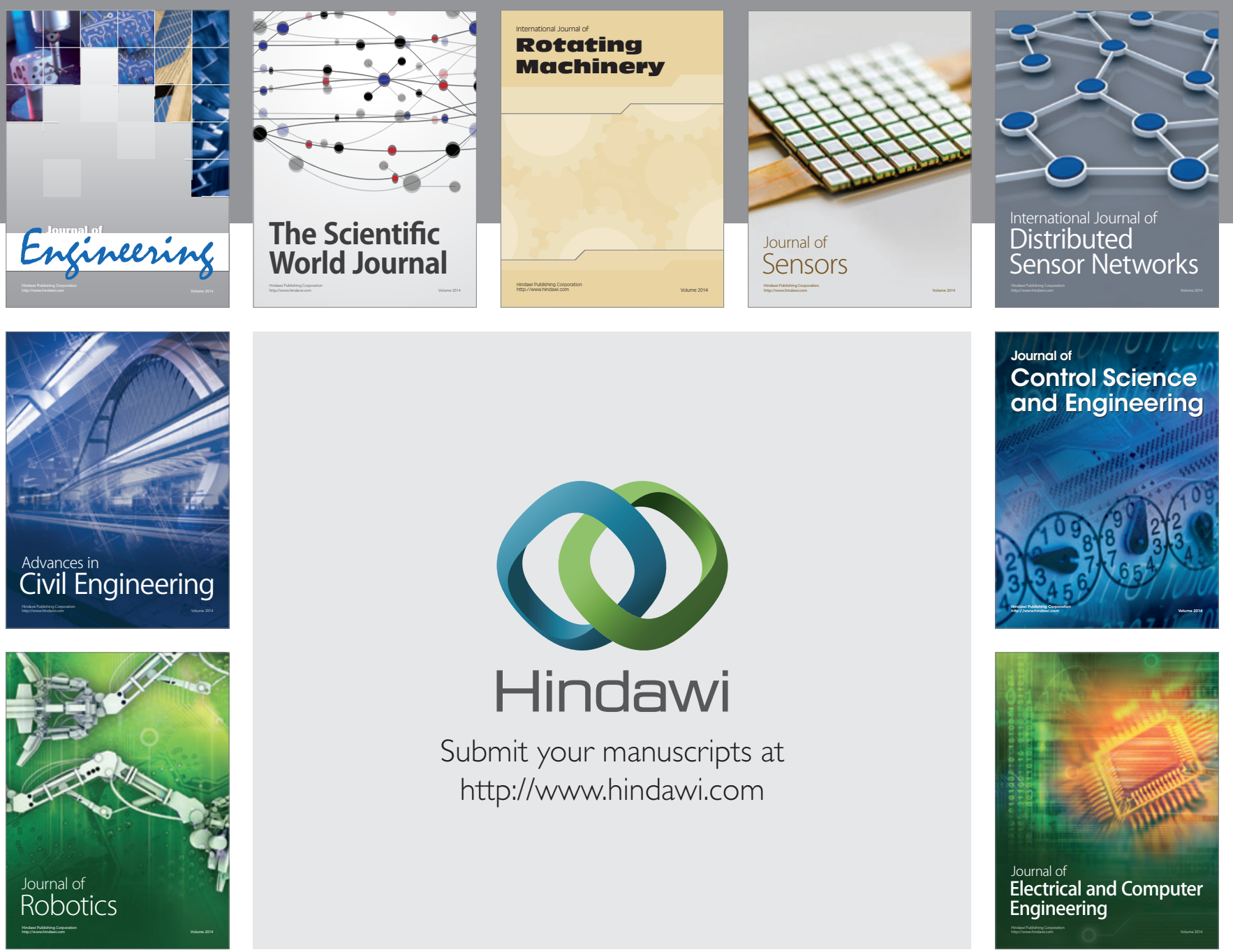

Submit your manuscripts at

http://www.hindawi.com
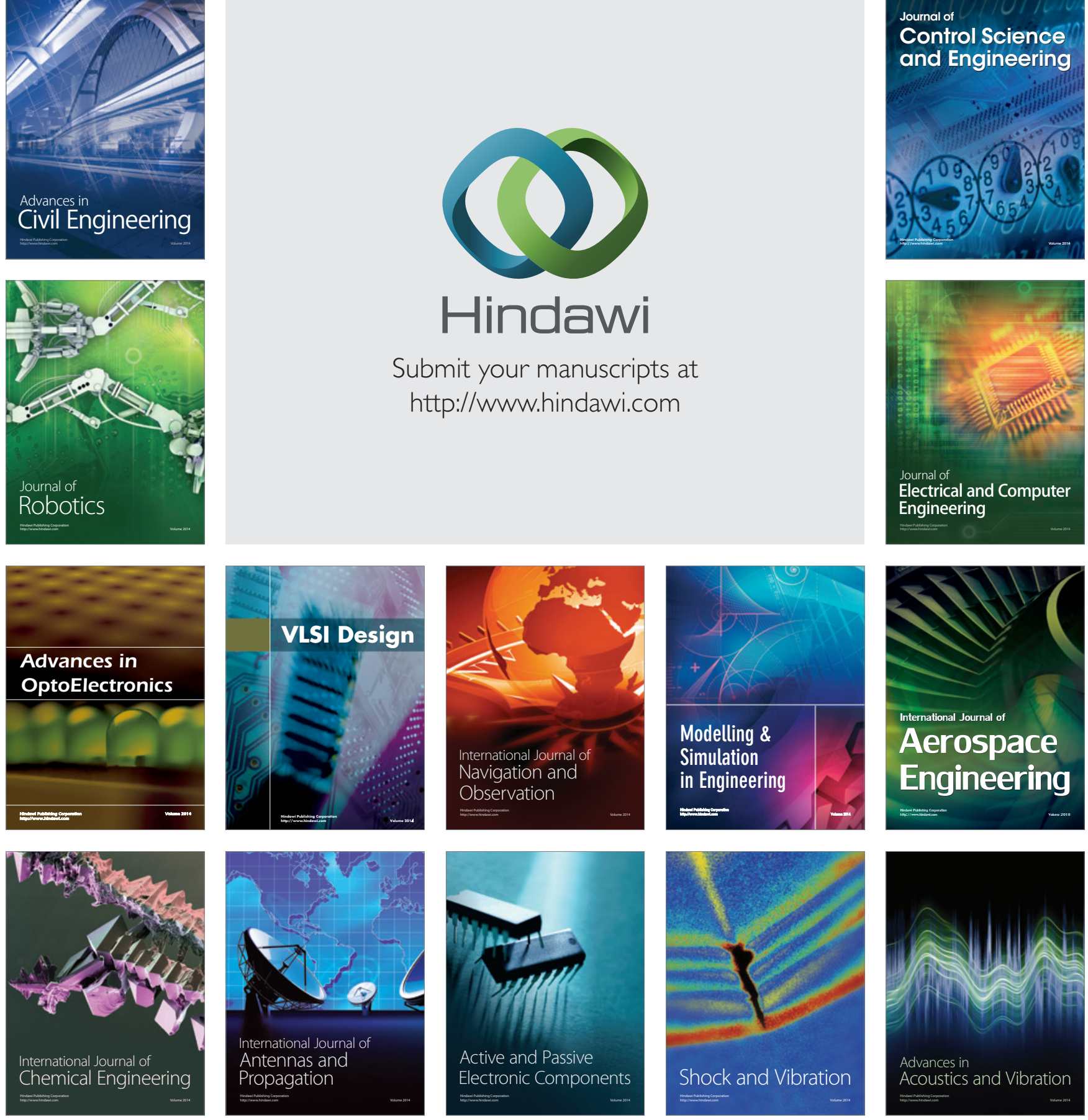